Provided for non-commercial research and education use. Not for reproduction, distribution or commercial use.

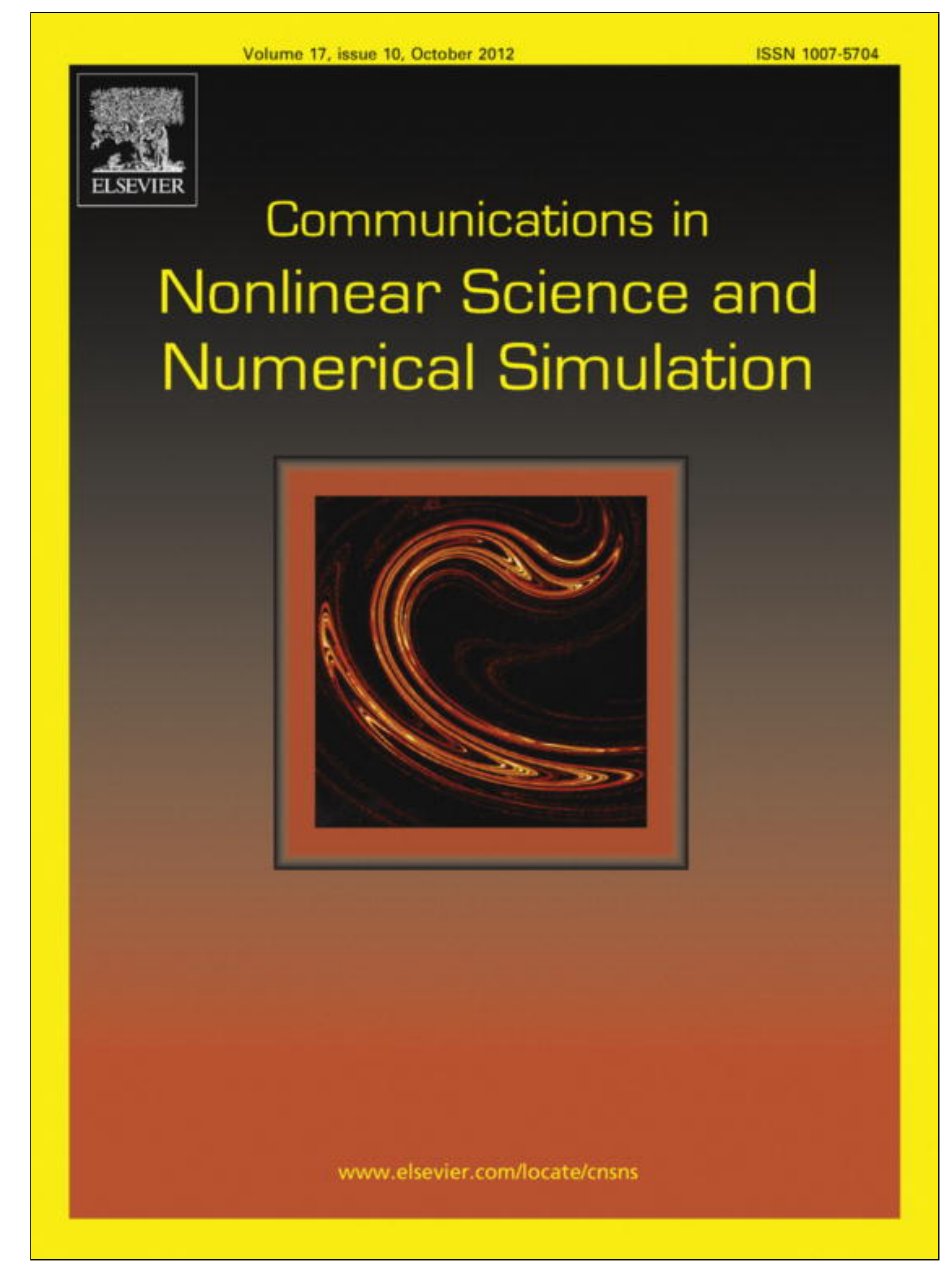

This article appeared in a journal published by Elsevier. The attached copy is furnished to the author for internal non-commercial research and education use, including for instruction at the authors institution and sharing with colleagues.

Other uses, including reproduction and distribution, or selling or licensing copies, or posting to personal, institutional or third party websites are prohibited.

In most cases authors are permitted to post their version of the article (e.g. in Word or Tex form) to their personal website or institutional repository. Authors requiring further information regarding Elsevier's archiving and manuscript policies are encouraged to visit:

http://www.elsevier.com/copyright 
Short communication

\title{
Homotopy analysis method with a non-homogeneous term in the auxiliary linear operator
}

\author{
Anant Kant Shukla ${ }^{\text {a,* }}$, T.R. Ramamohan ${ }^{\text {a }}$, S. Srinivas ${ }^{\mathrm{b}}$ \\ ${ }^{a}$ CSIR Centre for Mathematical Modelling and Computer Simulation, Council of Scientific and Industrial Research, Wind Tunnel Road, Bangalore 560 037, India \\ ${ }^{\mathrm{b}}$ VIT University, Vellore 632 014, India
}

\section{A R T I C L E I N F O}

\section{Article history:}

Received 11 September 2011

Received in revised form 11 February 2012

Accepted 13 February 2012

Available online 23 February 2012

\section{Keywords:}

Homotopy analysis method

Non-homogeneous auxiliary linear operator

Convergence theorem

Further generalization

\begin{abstract}
A B S T R A C T
We demonstrate the efficiency of a modification of the normal homotopy analysis method (HAM) proposed by Liao [2] by including a non-homogeneous term in the auxiliary linear operator (this can be considered as a special case of "further generalization" of HAM given by Liao in [2]). We then apply the modified method to a few examples. It is observed that including a non-homogeneous term gives faster convergence in comparison to normal HAM. We also prove a convergence theorem, which shows that our technique yields the convergent solution.
\end{abstract}

(c) 2012 Elsevier B.V. All rights reserved.

\section{Introduction}

Unlike perturbation methods [1], the homotopy analysis method (HAM) does not require the existence of a small parameter in terms of which a perturbation solution is developed and is thus valid for both weakly and strongly nonlinear problems. Most chemistry, engineering and physics problems are modelled by nonlinear differential equations. It is difficult to find analytical solutions of nonlinear problems except in a few examples. In recent years determining approximate analytical solutions using the homotopy analysis method has generated a lot of interest due to its applicability and efficiency and this technique has been successfully applied to a number of nonlinear problems [3-9]. Some modifications for different types of nonlinear equations have been developed in the literature [10-15]. Additional information on HAM can be found in Refs. [16-19]. In this paper we attempt to answer the question, "Is there any additional freedom we can exploit in the framework of the homotopy analysis method such that we get the same solutions in a fewer number of HAM approximations"? In other words, "Is it possible to reduce the order of the HAM approximation for obtaining the required approximate analytical solution to a given accuracy"? We give a brief introduction to Liao's scheme and the proposed scheme in the following two sections. Later we give the relation between the proposed scheme and a further generalization of Liao's scheme, as given in [2]. We then propose criteria for choosing the additional terms and solve the considered problems. Finally we give the conclusions of the major results of the paper.

\section{Basic idea of the homotopy analysis method}

Homotopy is a basic concept of topology [21,22]. To demonstrate the basic features of the homotopy analysis method, let us consider the following differential equation:

\footnotetext{
* Corresponding author. Tel.: +91 8025051943.

E-mail address: akshukla@cmmacs.ernet.in (A.K. Shukla).
} 


$$
N[u(t)]=0
$$

where $N$ is a nonlinear operator, $t$ denotes the independent variable and $u(t)$ is an unknown function. For simplicity we ignore all boundary and/or initial conditions, which can be treated in a similar manner. Liao [2] constructs the so-called zero order deformation equation as follows:

$$
(1-q) L\left[\phi(t ; q)-u_{0}(t)\right]=q h H(t) N[\phi(t ; q)]
$$

where $q \in[0,1]$ is the embedding parameter, $h$ is a nonzero auxiliary linear parameter, $H(t)$ is nonzero auxiliary function, $u_{0}(t)$ is the initial guess of $u(t), L$ is an auxiliary linear operator and $\phi(t ; q)$ is an unknown function. It is important, that one has a lot of freedom in choosing the auxiliary linear operator, auxiliary parameter and auxiliary function in the framework of the homotopy analysis method. Obviously, when $q=0$ and $q=1$, we have

$$
\phi(t ; 0)=u_{0}(t), \quad \phi(t ; 1)=u(t)
$$

respectively. Thus as $q$ increases from 0 to $1, \phi(t ; q)$ varies from the initial guess $u_{0}(t)$ to the exact solution $u(t)$. In topology this is called a deformation and $L\left[\phi(t ; 0)-u_{0}(t)\right]$ and $N[\phi(t ; 1)]$ are called homotopic. By expanding $\phi(t ; q)$ in a Taylor's series with respect to $q$, one has

$$
\phi(t ; q)=u_{0}(t)+\sum_{m=1}^{\infty} u_{m}(t) q^{m}
$$

where

$$
u_{m}(t)=\frac{1}{m !} \frac{\partial^{m} N[\phi(t ; q)]}{\partial q^{m}} \mid q=0
$$

If the initial guess, auxiliary linear operator, auxiliary parameter, and auxiliary function are properly chosen, such that the series (4) converges at $q=1$, then we have

$$
u(t)=u_{0}(t)+\sum_{m=1}^{\infty} u_{m}(t)
$$

which must be one of the solutions of the nonlinear equation as proved by Liao. According to (5) the governing equation can be found from the zero order deformation equation (2). For the sake of brevity, define the vector

$$
\vec{u}(t)=\left\{u_{0}(t), u_{1}(t), \ldots, u_{n}(t)\right\}
$$

Differentiating equation (2) $m$-times with respect to $q$ and then setting $q=0$ and finally dividing by $m$ !, we have the so-called $m$ th order deformation equation

$$
L\left[u_{m}(t)-\chi_{m} u_{m-1}(t)\right]=h H(t) R_{m}\left(u_{m-1}\right)
$$

where

$$
R_{m}\left(u_{m-1}\right)=\left.\frac{1}{(m-1) !} \frac{\partial^{m-1} N[\phi(t ; q)]}{\partial q^{m-1}}\right|_{q=0}
$$

and

$$
\chi_{m}= \begin{cases}0 ; & \text { for } m=1 \\ 1 ; & \text { for } m \neq 1\end{cases}
$$

\section{Non-homogeneous auxiliary linear operator}

To the best of our knowledge there is no prior implementation of the technique proposed in this work in the literature. In order to apply the HAM, a prior knowledge of some of the properties of the solution is desirable either through a knowledge of the physics or through numerical solutions, so that an appropriate choice of base functions can be made. After choosing the base functions we choose the auxiliary linear operator, auxiliary function and auxiliary parameter so that the solution of each deformation equation exists and the solution of each deformation equation can be expressed in terms of the base functions. Let (1) be the equation that we have to solve. Our technique modifies Liao's technique by taking the auxiliary linear operator in the following form:

$$
L[\phi(t ; q)]+c G(t ; q)
$$

where $L[\phi(t ; q)]$ is the usual auxiliary linear operator following Liao, $c$ is a constant and $G(t ; q)$ is a function of $t$ and $q$, which is zero at $q=0$ and $q=1$. For illustrating this general idea throughout the paper we choose a simple form of $G(t ; q)$, namely

$$
G(t ; q)=q(1-q) f(t)
$$


The form of the zero order deformation equation taken in this work is:

$$
(1-q) L\left[\phi(t ; q)-u_{0}(t)\right]+c q(1-q) f(t)=q h H(t) N[\phi(t ; q)]
$$

At, $c=0$ the above equation reduces to the original form (2) of the zero order deformation equation as taken by Liao [2]. After this change, the whole procedure of finding either the higher order deformation equations or the approximate solution is the same as mentioned previously. Applying this procedure may also reduce the computational cost for a given accuracy. At $q=0$ and $q=1$, Eq. (11) reduces to the form (3); this is the basic requirement of HAM. The procedure of finding the high order deformation equations is similar to Liao [20]. Only the form of the 1 st and 2 nd order deformation equation change, i.e., from 3rd order onwards, the form of the higher order deformation equations is exactly the same as Liao's [2]. Throughout the discussion the position of $c$ and $f(t)$ will be fixed and will be common to all problems. Clearly, at $c=0$ we obtain the solution of Liao's scheme.

\section{Relation to further generalization of HAM}

The above suggested approach can also be considered as a special case of "Further Generalization" of HAM given by Liao in his book [2]. Liao suggested a zeroth- order generalized deformation equation as:

$$
[1-B(q)] L\left[\phi(t ; q)-u_{0}(t)\right]=A(q) h H(t) N[\phi(t ; q)]+h_{2} H_{2}(t) \Pi[\phi(t ; q)]
$$

where $A(0)=B(0)=0$ and $A(1)=B(1)=1$ are convergent power series of $q$ in $[0,1]$ and

$$
\Pi[\phi(t ; 0)]=\Pi[\phi(t ; 1)]=0
$$

such as

$$
\begin{aligned}
& \Pi[\phi(t ; q)]=A(q)[1-B(q)] \mathbf{F}[\phi(t ; q)] \\
& \Pi[\phi(t ; q)]=[1-A(q)][\phi(t, q)]^{1+q}-\phi(t, q)
\end{aligned}
$$

We can relate our approach to Liao's generalized form as a special case by choosing $A(q)=B(q)=q, H_{2}(t)=1, h_{2}=-1$ and $\mathbf{F}=f(t)$.

\section{Criteria for the choice of $\boldsymbol{c}$ and $\boldsymbol{f}(\boldsymbol{t})$}

Let $\left\{e_{k}(t): k=1,2,3, \ldots\right\}$ denote the complete set of base function for the solution of $u(t)$. Then, we can express

$$
f(t)=\sum_{k=1}^{s} c_{k} e_{k}(t)
$$

where $s$ is a positive integer and the constant " $c$ " is presumed to be subsumed in the constants " $c_{k}$ ". Then, the $m$ th order HAM approximation contains $(s+1)$ convergence-control parameters namely: $h, c_{1}, c_{2}, \ldots, c_{s}$, whose optimal value can be determined by the minimization of the square residual error of the governing equation as shown by Liao in the so-called optimal HAM [15]. Suppose $\Delta_{m}$ is the square residual error of Eq. (1) at $m$ th order HAM approximation, then

$$
\Delta_{m}=\int_{a}^{b}\left(N\left[\sum_{i=0}^{m} u_{i}(\xi)\right]\right)^{2} d \xi
$$

where the interval $[a, b]$ belongs to the domain of the problem considered. We can determine the constants by solving the following algebraic equations:

$$
\frac{\partial \Delta_{m}}{\partial h}=0, \frac{\partial \Delta_{m}}{\partial c_{1}}=0, \ldots
$$

\section{Mathematical formulation, convergence theorem and examples}

In order to demonstrate the efficiency of this technique we present an analysis of the following problems and also prove a convergence theorem.

Example 1. Consider the following non-linear differential equation [2]:

$$
u^{\prime}(t)+u^{2}(t)=1, \quad u(0)=0, t \geqslant 0
$$

The exact solution of Eq. (19) is $\tanh (t)$. Following Liao [2], we choose exponential base functions, with the same initial guess of $u(t)$, i.e.,

$$
u_{0}(t)=1-\exp (-t)
$$


Choose the non-homogeneous auxiliary linear operator as follows:

$$
L[\phi(t ; q)]+c q(1-q) f(t)
$$

where

$$
L[\phi(t ; q)]=\frac{\partial[\phi(t ; q)]}{\partial t}+\phi(t ; q)
$$

From Eq. (19) we define the nonlinear operator as:

$$
N[\phi(t ; q)]=\phi^{\prime}(t ; q)+\phi^{2}(t ; q)-1
$$

The high-order deformation equation is

$$
L\left[u_{m}(t)-\chi_{m} u_{m-1}(t)\right]+c g(t)=h H(t) R_{m}\left(u_{m}\right)
$$

where

$$
g(t)= \begin{cases}f(t) ; & \text { for } m=1 \\ -f(t) ; & \text { for } m=2 \\ 0 ; & \text { for } m \geqslant 3\end{cases}
$$

Therefore the $m$ th order deformation equations are

$$
\begin{aligned}
& L\left[u_{1}(t)\right]=h H(t) R_{1}(t)-c f(t), \quad m=1 \\
& L\left[u_{2}(t)-u_{1}(t)\right]=h H(t) R_{2}(t)+c f(t), \quad m=2 \\
& L\left[u_{m}(t)-u_{m-1}(t)\right]=h H(t) R_{m}(t), \quad m \geqslant 3
\end{aligned}
$$

subject to the initial conditions:

$$
u_{m}(t)=0, \quad m \geqslant 1
$$

where

$$
R_{m}\left(u_{m-1}\right)=u_{m-1}^{\prime}+\sum_{n=0}^{m-1} u_{n}(t) u_{m-n-1}(t)-\left[1-\chi_{m}\right]
$$

The $m$ th order approximate solution $u(t)$ is given by:

$$
u(t)=\sum_{m=0}^{\infty} u_{m}(t)
$$

Before discussing the solution, following Liao [2], we prove a convergence theorem.

Theorem 1. As long as the series (30) converges, where $u_{m}(t)$ is governed by the high-order deformation equations (25)-(28) under the definition (10) and (29), it must be the exact solution of (19).

Proof. If the series

$$
u(t)=\sum_{m=0}^{\infty} u_{m}(t)
$$

converges, we can write

$$
S(t)=\sum_{m=0}^{\infty} u_{m}(t)
$$

and it holds,

$$
\lim _{m \rightarrow \infty} u_{m}(t)=0
$$

From Eqs. (25)-(27) using (10) successively we get the following equations for $m=1,2,3, \ldots, n$

$$
\begin{aligned}
& L\left[u_{1}(t)\right]=h H(t) R_{1}(t)-c f(t) \\
& L\left[u_{2}(t)-u_{1}(t)\right]=h H(t) R_{2}(t)+c f(t), \\
& \vdots \\
& L\left[u_{n}(t)-u_{n-1}(t)\right]=h H(t) R_{n}(t),
\end{aligned}
$$


Now adding column-wise we get

$$
L\left[u_{1}+\left(u_{2}-u_{1}\right)+\cdots+\left(u_{n}-u_{n-1}\right)\right]=\left(h H(t) R_{1}-c f(t)\right)+\left(h H(t) R_{2}+c f(t)\right)+\cdots+h(t) H(t) R_{n}
$$

i.e.,

$$
L\left[u_{n}\right]=h H(t) \sum_{m=1}^{n} R_{m}
$$

Taking the limit $n \rightarrow \infty$ and noting that $h \neq 0$ and $H(t) \neq 0$ and finally using (31), we get

$$
\sum_{m=1}^{\infty} R_{m}(t)=0
$$

From (29) we have

$$
\begin{aligned}
\sum_{m=1}^{\infty} R_{m}(t) & =\sum_{m=1}^{\infty}\left[u_{m-1}^{\prime}(t)+\sum_{n=0}^{m-1} u_{n}(t) u_{m-n-1}(t)-\left(1-\chi_{m}\right)\right]=\sum_{m=0}^{\infty} u_{m}^{\prime}-1+\sum_{m=1}^{\infty} \sum_{n=0}^{m-1} u_{n}(t) u_{m-n-1}(t) \\
& =\sum_{m=0}^{\infty} u_{m}^{\prime}-1+\sum_{n=0}^{\infty} \sum_{m=n+1}^{\infty} u_{n}(t) u_{m-n-1}(t)=\sum_{m=0}^{\infty} u_{m}^{\prime}-1+\sum_{n=0}^{\infty} u_{n}(t) \sum_{i=0}^{\infty} u_{i}(t)=\dot{S}(t)+S^{2}(t)-1
\end{aligned}
$$

From the above equations we have

$$
\dot{S}(t)+S^{2}(t)-1=0 ; \quad t \geqslant 0
$$

and it also holds

$$
S(0)=0
$$

Therefore according to the above two equations $S(t)$ must be the exact solution of (19). Thus the theorem is valid even in the case of the non-homogeneous auxiliary linear operator. It is evident from the proof that this is true for any value of $c$ and $f(t)$.

\section{Solution:}

We choose $f(t)=c_{1} \exp (-t)+c_{2} \exp (-2 t)$ and fix $c_{1}=0$ because it's use would produce secular terms. We fix $h=-1$ and $H(t)=\exp (-t)$ for both the schemes. We can determine $c_{2}$ from the Fig. 1 where $\Delta_{m}$ has been plotted vs. $c_{2}$. For $c_{2}=0.06$ the $h$-curves are presented in Fig. 2 and for Liao's scheme (where $c_{1}=c_{2}=0$ ) $h$-curves are presented in Fig. 3, the corresponding solutions at different orders of HAM approximation and the corresponding series of derivatives from both the schemes are presented in Tables 1 and 2. The solution expression for both the schemes of order 10 with $h=-1$ are given in Eqs. (39) and (40):

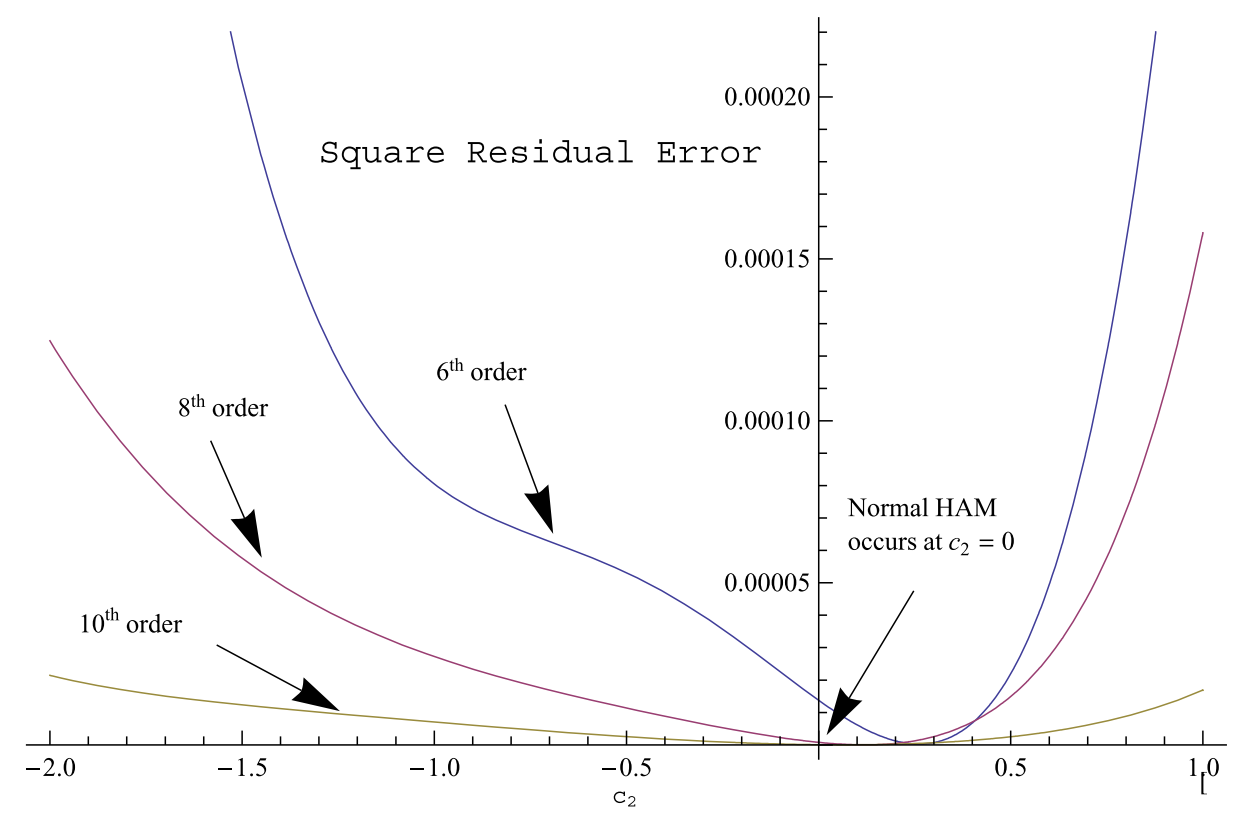

Fig. 1. Square residual error $\Delta_{m}$ vs. $c_{2}$ in case $h=-1, c_{1}=0$ of order $6,8,10$, by means of $H(t)=\exp (-t)$. Blue: 6 th order; red: 8 th order; yellow: 10 th order. (For interpretation of the references to colour in this figure legend, the reader is referred to the web version of this article.) 


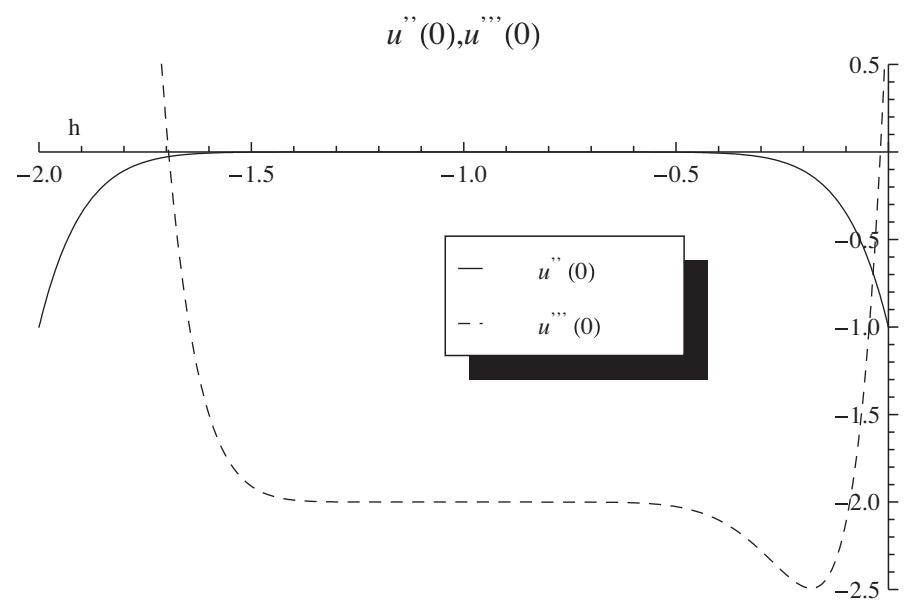

Fig. 2. $h$-Curves found by Liao's scheme; solid line: $u^{\prime \prime}(0)$ vs. $h$; dashed line: $u^{\prime \prime \prime}(0)$ vs. $h$ of order 10 , by means of $H(t)=\exp (-t)$.

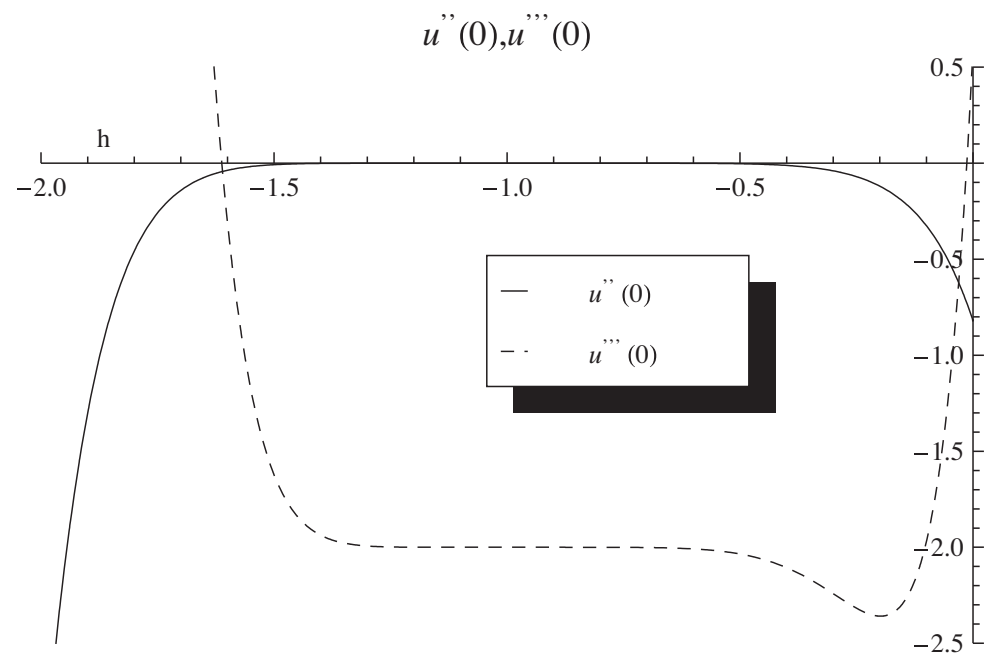

Fig. 3. $h$-Curves found by proposed scheme with $c_{2}=0.06$ and $f(t)=\exp (-2 t)$; solid line: $u^{\prime \prime}(0)$ vs. $h$; dashed line: $u^{\prime \prime \prime}(0)$ vs. $h$ of order 10 , by means of $H(t)=\exp (-t)$.

Table 1

Comparison between Liao's scheme and proposed scheme with exact solution. We have taken $h=-1$ and $H(t)=\exp (-t)$ for both the schemes.

\begin{tabular}{|c|c|c|c|c|c|}
\hline$t$ & HAM 5th order & Proposed 5th order & HAM 10 th order & Proposed 10th order & Exact solution \\
\hline $1 / 4$ & 0.24491 & 0.24490 & 0.24492 & 0.24492 & 0.24492 \\
\hline $1 / 2$ & 0.46190 & 0.46184 & 0.46212 & 0.46212 & 0.46212 \\
\hline $3 / 4$ & 0.63420 & 0.63414 & 0.63514 & 0.63515 & 0.63515 \\
\hline 1 & 0.75964 & 0.75965 & 0.76156 & 0.76159 & 0.76159 \\
\hline $3 / 2$ & 0.90204 & 0.90225 & 0.90507 & 0.90513 & 0.90514 \\
\hline 2 & 0.96120 & 0.96147 & 0.96395 & 0.96402 & 0.96403 \\
\hline $5 / 2$ & 0.98454 & 0.98477 & 0.98655 & 0.98661 & 0.98661 \\
\hline 3 & 0.99367 & 0.99384 & 0.99501 & 0.99506 & 0.99505 \\
\hline 4 & 0.99878 & 0.99885 & 0.99931 & 0.99933 & 0.99933 \\
\hline 5 & 0.99970 & 0.99973 & 0.99990 & 0.99991 & 0.99991 \\
\hline 10 & 1.00000 & 1.00000 & 1.00000 & 1.00000 & 1.00000 \\
\hline 100 & 1.00000 & 1.00000 & 1.00000 & 1.00000 & 1.00000 \\
\hline
\end{tabular}

$$
\begin{aligned}
u_{\text {Liao }}(t)= & 1-\frac{e^{-21 t}}{1024}+\frac{e^{-20 t}}{512}+\frac{5 e^{-19 t}}{512}-\frac{11 e^{-18 t}}{512}-\frac{45 e^{-17 t}}{1024}+\frac{15 e^{-15 t}}{128}-\frac{11 e^{-14 t}}{32}-\frac{105 e^{-13 t}}{512}+\frac{193 e^{-12 t}}{256}+\frac{63 e^{-11 t}}{256}-\frac{319 e^{-10 t}}{256} \\
& -\frac{105 e^{-9 t}}{512}+\frac{7 e^{-16 t}}{64}+\frac{53 e^{-8 t}}{32}+\frac{15 e^{-7 t}}{128}-\frac{121 e^{-6 t}}{64}-\frac{45 e^{-5 t}}{1024}+\frac{1013 e^{-4 t}}{512}+\frac{5 e^{-3 t}}{512}-\frac{1023 e^{-2 t}}{512}-\frac{e^{-t}}{1024}
\end{aligned}
$$


Table 2

Comparison between the corresponding first derivative series for both the schemes with first derivative of exact solution at different time.

\begin{tabular}{llll}
\hline$t$ & Derivative of $(39)$ & Derivative of $(40)$ & Derivative of $\tanh (t)$ \\
\hline 0 & 1.00000000 & 1.00000000 & 1.00000000 \\
1 & 0.41990000 & 0.41990000 & 0.42000000 \\
2 & 0.07067000 & 0.07067000 & 0.07067000 \\
3 & 0.00990200 & 0.00986800 & 0.00986600 \\
4 & 0.00135700 & 0.000134000 & 0.00134100 \\
5 & 0.00018800 & 0.00002419 & 0.00018160 \\
6 & 0.00002697 & 0.00002458 \\
\hline
\end{tabular}

$$
\begin{aligned}
u_{\text {proposed }}(t)= & -\frac{e^{-21 t}}{1024}+\frac{83 e^{-20 t}}{64,000}+\frac{71,557 e^{-19 t}}{6,400,000}-\frac{5,175,155,837 e^{-18 t}}{308,000,000,000}-\frac{691,352,811,153 e^{-17 t}}{12,320,000,000,000} \\
& +\frac{145,445,517,892,193 e^{-16 t}}{1,501,500,000,000,000}+\frac{340,952,708,043,601 e^{-15 t}}{2,102,100,000,000,000}-\frac{12,461,231,893,639 e^{-14 t}}{37,537,500,000,000} \\
& -\frac{2,756,573,215,259 e^{-13 t}}{9,240,000,000,000}+\frac{29,492,523,646,261 e^{-12 t}}{38,500,000,000,000}+\frac{3,815,504,752,991 e^{-11 t}}{10,500,000,000,000} \\
& -\frac{336,389,835,907 e^{-10 t}}{262,500,000,000}-\frac{959,889,392,353 e^{-9 t}}{2,800,000,000,000}+\frac{8,939,444,197,793 e^{-8 t}}{4,900,000,000,000} \\
& -\frac{2,448,652,614,491 e^{-7 t}}{115,500,000,000,000}-\frac{10,030,822,156,459 e^{-6 t}}{5,775,000,000,000}-\frac{1,639,774,008,067 e^{-5 t}}{9,240,000,000,000} \\
& +\frac{376,214,885,173 e^{-4 t}}{184,800,000,000}+\frac{1,235,490,931 e^{-3 t}}{223,437,500,000}-\frac{3,007,101,185,274,421 e^{-2 t}}{1,501,500,000,000,000} \\
& +\frac{14,222,289,524,479 e^{-t}}{84,084,000,000,000,000}
\end{aligned}
$$

Example 2. Consider the nonlinear differential equation called the Duffing oscillator in space, see reference [2]:

$$
v^{\prime \prime}(t)+\epsilon\left(v(t)-v^{3}(t)\right)=0
$$

with the boundary conditions:

$$
v(0)=v(\pi)=0
$$

\section{Solution:}

\section{Define}

$$
A=v\left(\frac{\pi}{2}\right), \quad v(t)=A u(t)
$$

then Eq. (41) becomes

$$
u^{\prime \prime}(t)+\epsilon\left(u(t)-A^{2} u^{3}(t)\right)=0, \quad u(0)=u(\pi)=0
$$

It is obvious that

$$
u\left(\frac{\pi}{2}\right)=1
$$

According to Kahn and Zarmi [23], the exact relation between $\epsilon$ and $A$ is:

$$
\epsilon=\left[\frac{2}{\pi \sqrt{1-\frac{A^{2}}{2}}} K\left(\frac{A^{2}}{2-A^{2}}\right)\right]^{2}
$$

where $K(\zeta)$ is the complete elliptic integral of first kind. According to the above exact solution, $\epsilon$ tends to infinity as $|A|$ approaches to 1 . We find the amplitude $A$ using the proposed scheme by removing the secular terms [2] and then we compare our results with Liao's results [2]. We choose the same initial guess $u_{0}(t)=\sin (t)$ and the auxiliary function $H(t)=1$ for both the schemes. The set of base functions in this case is

$$
\{\sin [(2 m+1) t]: m \geqslant 0\}
$$

and the auxiliary linear operator is

$$
L[\phi(t ; q)]=\frac{\partial^{2}[\phi(t ; q)]}{\partial t^{2}}+\phi(t ; q)
$$


This is the auxiliary operator in Liao's scheme. We apply the above mentioned procedure for this problem. We choose $f(t)=c_{1} \sin (t)+c_{2} \sin (3 t)+c_{3} \sin (5 t)$ and fix $c_{1}=0$ because it's use would produce secular terms. We fix $h=-\frac{1}{5}$ and $H(t)=1$ for both the schemes. As a special case we use $c_{2}=0$ and find $c_{3}$ from Fig. 4 . The corresponding results are presented in Table 3. Following Liao [2], we take

$$
h=-\frac{1}{\left(1+\frac{\epsilon}{3}\right)}
$$

then for each value of $\epsilon$ we obtain the square residual error in Fig. 5 and we choose $c_{1}=0$ for $f(t)=c_{1} \sin (t)+c_{2} \sin (3 t)$. Clearly, the square residual error decreases. We find approximately the same h-curves as found by Liao [2], so that the region of convergence is approximately the same.

Example 3. For the above example it has been shown in [2] that it admits multiple solutions, when the set of base functions is of the form

$$
\{\sin [(2 m+1) \kappa t]: m \geqslant 0, \kappa \geqslant 1\}
$$

Following Liao [2], we determine the multiple solutions including the non-homogeneous term. Without loss of generality, define

$$
A=v\left(\frac{\pi}{2 \kappa}\right), \quad v(t)=A u(t)
$$

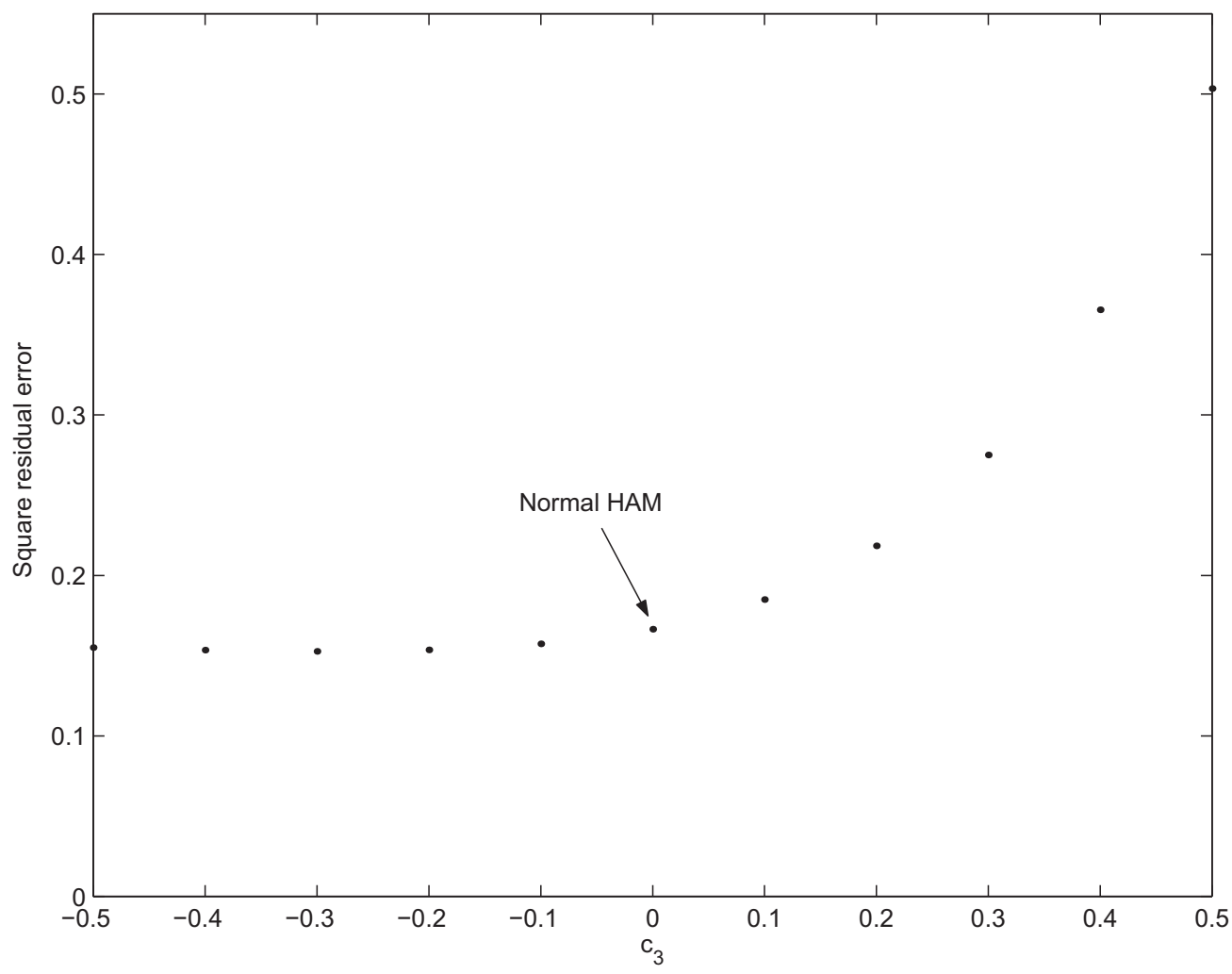

Fig. 4. Square residual error $\Delta_{m}$ vs. $c_{3}$ in case $\epsilon=25, h=-\frac{1}{5}, c_{1}=c_{2}=0$ of order 11 .

Table 3

The analytical approximations of $A$ when $\epsilon=25, h=-\frac{1}{5}$ and $H(t)=1$.

\begin{tabular}{lll}
\hline $\begin{array}{l}\text { Order of } \\
\text { approximation }\end{array}$ & $\begin{array}{l}\epsilon=25 \text { by Liao's } \\
\text { scheme }\end{array}$ & $\begin{array}{l}\epsilon=25, c_{2}=-0.3, \\
f(t)=\sin (5 t) \text { by proposed } \\
\text { scheme }\end{array}$ \\
\hline 5 & 1.01046 & 0.99642 \\
10 & 1.00313 & 1.00169 \\
15 & 1.00117 & 1.00044 \\
20 & 1.00049 & 1.00014 \\
25 & 1.00017 & 1.00003 \\
\hline
\end{tabular}




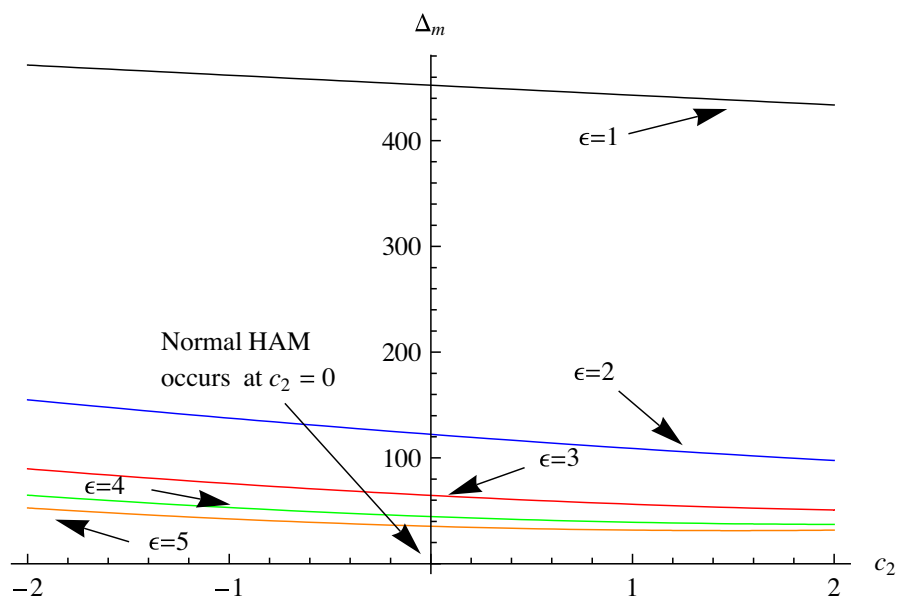

Fig. 5. Square residual error $\Delta_{m}$ vs. $c_{2}$ in case $\epsilon=1,2,3,4,5 ; h=-\frac{1}{\left(1+\frac{\epsilon}{3}\right)}, c_{1}=0$ of order 3; black: $\epsilon=1$; blue: $\epsilon=2$; red: $\epsilon=3$; green: $\epsilon=4$; orange: $\epsilon=5$; vertical line corresponds the residual error for Liao's scheme for each $\epsilon$. (For interpretation of the references to colour in this figure legend, the reader is referred to the web version of this article.)

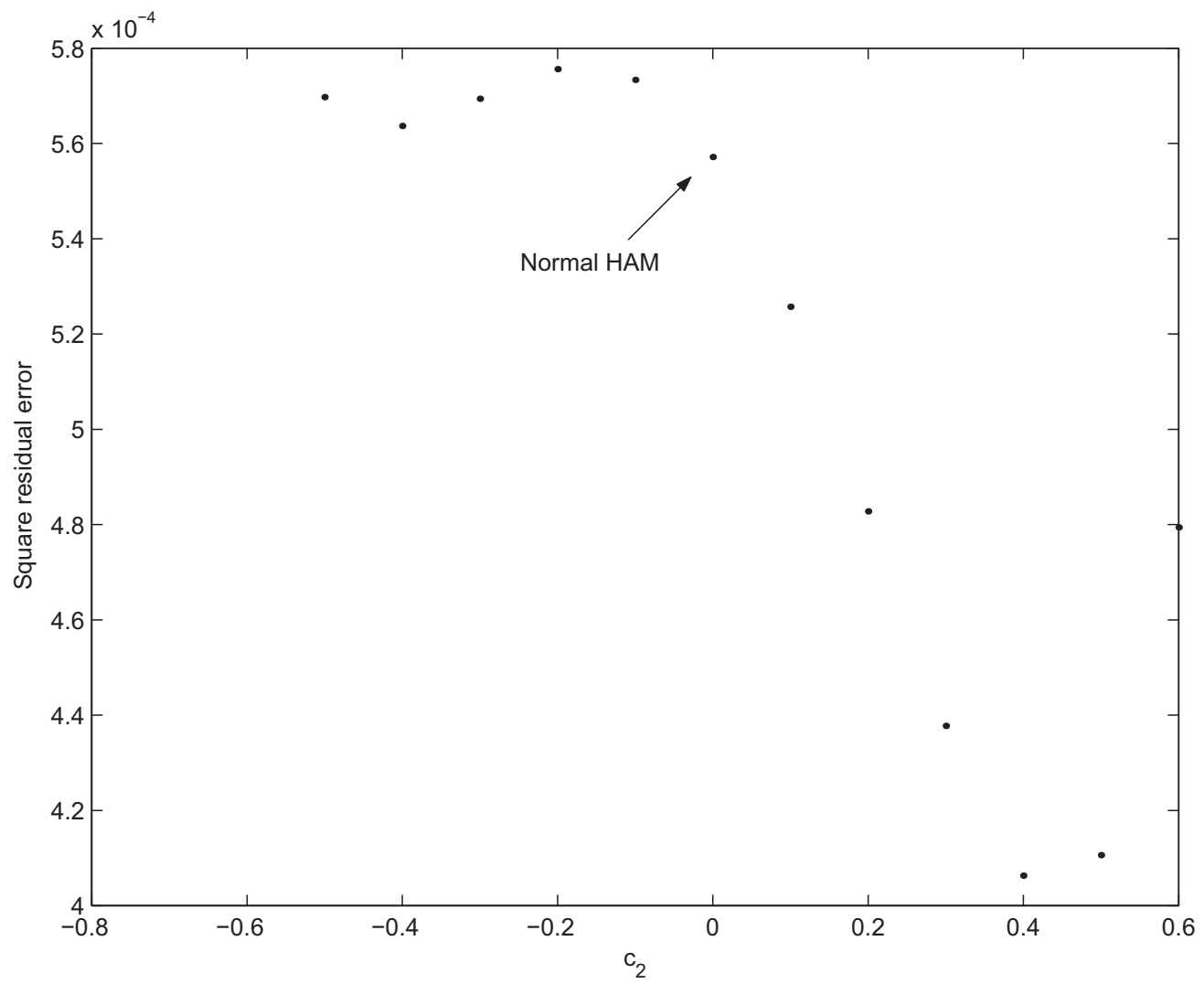

Fig. 6. Square residual error $\Delta_{m}$ vs. $c_{2}$ in case $h=-\frac{1}{2}, c_{1}=0, \kappa=2, \epsilon=40$ of order 11 , by means of $H(t)=1$.

then Eq. (41) becomes

$$
u^{\prime \prime}(t)+\epsilon\left(u(t)-A^{2} u^{3}(t)\right)=0, \quad u(0)=u(\pi)=0
$$

Clearly $A$ is unknown in the above equation and we obtain find this by removing the secular terms like [2] for different values of $\epsilon$ and $\kappa$. From Eq. (50), it holds

$$
u\left(\frac{\pi}{2 \kappa}\right)=1
$$

Because the problem is the same except for the parameter $\kappa$, we can apply the above technique here also. We choose the same initial guess $u_{0}(t)=\sin (\kappa t)$ and the auxiliary function $H(t)=1$ as taken by Liao in [2]. In this case we choose 
$f(t)=c_{1} \sin (2 t)+c_{2} \sin (6 t)$ and $f(t)=c_{1} \sin (3 t)+c_{2} \sin (9 t)$ for $\kappa=2$ and $\kappa=3$, respectively. We fix $c_{1}=0, h=-\frac{1}{2}$ and $H(t)=1$. We find $c_{2}$ from Figs. 6 and 7 for $\kappa=2$ and $\kappa=3$, respectively. We present a comparison between both the schemes in Tables 4 and 5 . The exact analytical result is given by the implicit formula

$$
\epsilon=\frac{8 \kappa^{2}}{\pi^{2}\left(2-A^{2}\right)} K\left(\frac{A^{2}}{2-A^{2}}\right)
$$

where $K(\zeta)$ is the complete elliptic integral of first kind. For $\epsilon=40, \kappa=2$ and $c_{2}=2 / 5$, the solution expression of $u(t)$ of order 5 at $h=-1 / 2$ by means of $H(t)=1$ is given in Eq. (54). Similarly $\epsilon=90, \kappa=3$ and $c=1.2$, the solution expression of $u(t)$ of order 5 at $h=-\frac{1}{2}$ by means of $H(t)=1$ is given in Eq. (55):

$$
\begin{aligned}
u_{(\text {proposed }, \epsilon=40)}(t)= & 1.1764 \sin (2 t)+0.21834 \sin (6 t)+0.053345 \sin (10)+0.013777 \sin (14 t)+0.0025753 \\
& \times \sin (18 t)+0.00020878 \sin (22 t)
\end{aligned}
$$

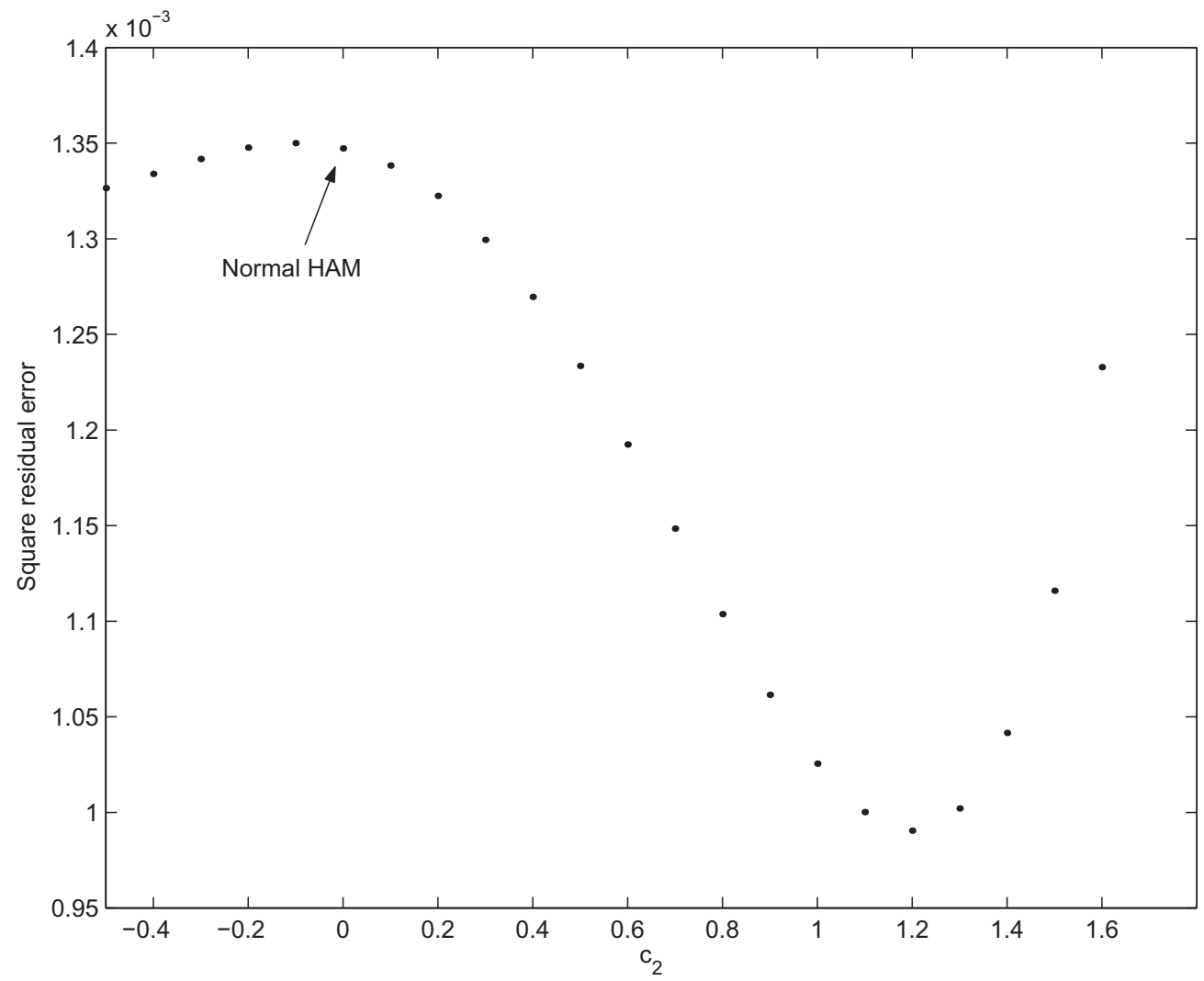

Fig. 7. Square residual error $\Delta_{m}$ vs. $c_{2}$ in case $h=-\frac{1}{2}, c_{1}=0, \kappa=3, \epsilon=90$ of order 11 , by means of $H(t)=1$.

Table 4

Comparison of the analytical approximation of $A$ between Liao's scheme and proposed scheme when $\epsilon=40, \kappa=2$ by means of $h=-\frac{1}{2}$ and $H(t)=1$.

\begin{tabular}{lll}
\hline Order of approximation & $\epsilon=40, \kappa=2$ Liao's scheme & $\begin{array}{l}\epsilon=40, \kappa=2, c_{2}=2 / 5, \\
f(t)=\sin (6 t) \text { proposed scheme }\end{array}$ \\
\hline 2 & 0.98070 & 0.97788 \\
4 & 0.99912 & 1.00064 \\
6 & 0.99613 & 0.99524 \\
8 & 0.99656 & 0.99683 \\
10 & 0.99656 & 0.99632 \\
12 & 0.99635 & 0.99646 \\
14 & 0.99649 & 0.99644 \\
16 & 0.99641 & 0.99643 \\
18 & 0.99645 & 0.99644 \\
20 & 0.99643 & 0.99643 \\
22 & 0.99644 & 0.99644 \\
\hline
\end{tabular}


Table 5

Comparison of the analytical approximation of $A$ between Liao's scheme and proposed scheme when $\epsilon=90, \kappa=3$ by means of $h=-\frac{1}{2}$ and $H(t)=1$.

\begin{tabular}{ccl}
\hline Order of approximation & $\epsilon=90, \kappa=3$ Liao's scheme & $\begin{array}{l}\epsilon=90, \kappa=3, c_{2}=1.2, \\
f(t)=\sin (9 t) \text { proposed scheme }\end{array}$ \\
\hline 2 & 0.98170 & 0.97792 \\
4 & 0.99854 & 1.0005 \\
6 & 0.99639 & 0.99528 \\
8 & 0.99625 & 0.99682 \\
10 & 0.99658 & 0.99632 \\
12 & 0.99635 & 0.99646 \\
14 & 0.99648 & 0.99643 \\
16 & 0.99642 & 0.99643 \\
18 & 0.99644 & 0.99644 \\
20 & 0.99643 & 0.99643 \\
22 & 0.99643 & 0.99644 \\
\hline &
\end{tabular}

\section{Conclusion}

It is observed from Figs. 2 and 3, that the non-homogeneous term does not effect the region of convergence of the problems considered so far. However the use of the non-homogeneous term helps in reducing the square residual error, as clear from Figs. 1 and 4-7. It is clear from Table 1, that we obtain adequate accuracy with our proposed scheme for a maximum approximation of order 10. This is a significant improvement over Liao's scheme with a little additional effort. The first derivative of the approximate solution found by the proposed scheme also agrees well with the first derivative of the exact solution in comparison to Liao's scheme as shown in Table 2. Similarly, we see faster convergence not only for the solutions but also for the HAM amplitudes found by removing the secular term for the Duffing-oscillator problem in space. This is clear from Tables 3-5. We can observe from the above analysis that a little additional effort leads to a significant improvement in the approximate solutions by reducing the square residual error.

\section{Acknowledgements}

The authors thank the Council of Scientific and Industrial Research (CSIR), India, for financial support and Prof. P. Seshu, Scientist-in-Charge, CSIR-CMMACS, for constant encouragement. We also wish to acknowledge the referee for comments which helped.

\section{References}

[1] Nayfeh AH. Perturbation methods. New York: John Wiley and Sons; 2000.

[2] Liao SJ. Beyond perturbation: an introduction to the homotopy analysis method. Boca Raton: CRC Press, Chapman and Hall; 2003.

[3] Allan FM, Syam MI. On the analytic solutions of the nonhomogeneous Blasius problem. J Comput Appl Math 2005;182:362-71.

[4] Abbasbandy S. The application of the homotopy analysis method to nonlinear equations arising in heat transfer. Phys Lett A 2006;360:109-13.

[5] Hang Xu, Liao SJ, Pop I. Series solution of unsteady boundary layer flows of non-newtonian fluid near a forward stagnation point. J Non-Newtonian Fluid Mech 2006;139:31-43.

[6] Bataineh AS, Noorani MSM, Hashim I. Approximate analytical solutions of system of PDEs by homotopy analysis method. Comput Math Appl 2008;55:2913-23.

[7] Sajid M, Awais M, Nadeem S, Hayat T. The influence of slip condition on thin film flow of a fourth grade fluid by the homotopy analysis method. Comput Math Appl 2008;56:2019-26.

[8] Hayat T, Abbas Z, Pop I, Asghar S. Effects of radiation and magnetic field on the mixed convection stagnation-point flow over a vertical stretching sheet in a porous medium. Int J Heat Mass Transf 2010;53:466-74.

[9] Srinivas S, Muthuraj R. Effects of thermal radiation and space porosity on MHD mixed convection flow in a vertical channel using homotopy analysis method. Commun Nonlinear Sci Numer Simulat 2010;15:2098-108.

[10] Bataineh AS, Noorani MSM, Hashim I. On a new reliable modification of homotopy analysis method. Commun Nonlinear Sci Numer Simulat 2009;14:409-23.

[11] Bataineh AS, Noorani MSM, Hashim I. Modified homotopy ananlysis method for solving system of second-order BVPs. Commun Nonlinear Sci Numer Simulat 2009;14:430-42.

[12] Odibat Z, Momani S, Hang Xu. A reliable algorithm of homotopy analysis method for solving nonlinear fractional differential equations. Appl Math Model 2010;34:593-600.

[13] Alomari AK, Noorani MSM, Nazar R. Homotopy approach for the hyperchaotic Chen system. Phys Scr 2010;81:045005 (7pp.).

[14] Hasan HN, El-Tawil MA. A new technique of using homotopy analysis method for solving high-order nonlinear differential equations. Math Methods ApplSci 2011;34:728-42.

[15] Liao SJ. An optimal homotopy analysis approach for strongly nonlinear differential equations. Commun Nonlinear Sci Numer Simulat 2010;15:2003-16.

[16] Liao SJ. On the homotopy analysis method for nonlinear problems. Appl Math Comput 2004;147:499-513. 
[17] Liao SJ, Tan Y. A general approach to obtain series solution of nonlinear differential equations. Stud Appl Math 2007;119:297-354.

[18] Abbasbandy S, Shivaniana E, Vajravelu K. Mathematical properties of h-curve in the frame work of the homotopy analysis method. Commun Nonlinear Sci Numer Simulat 2011;16:4268-75.

[19] Liao SJ. Comparison between the homotopy analysis method and homotopy perturbation method. Appl Math Comput 2005;169:1186-94.

[20] Liao SJ. Notes on the homotopy analysis method: some definitions and theorems. Commun Nonlinear Sci Numer Simulat 2009;14:983-97.

[21] Hilton PJ. An introduction to homotopy theory. Cambridge University Press; 1953.

[22] Sen S. Topology and geometry for physicists. Florida: Academic Press; 1983.

[23] Kahn PB, Zarmi Y. Nonlinear dynamics: exploration through normal forms. New York: John Wiley \& Sons Inc.; 1998. 